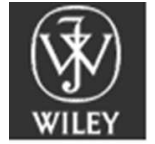

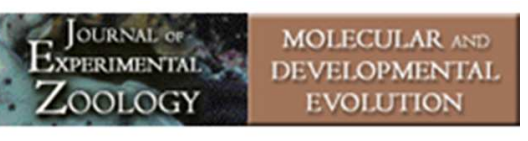

\title{
Cell proliferation dynamics in regeneration of the operculum head appendage in the annelid Pomatoceros lamarckii.
}

\begin{tabular}{|r|l|}
\hline Journal: & JEZ Part B: Molecular and Developmental Evolution \\
\hline Manuscript ID: & JEZ-B-2013-11-0087.R1 \\
\hline Wiley - Manuscript type: & Research Article \\
\hline Date Submitted by the Author: & n/a \\
\hline Complete List of Authors: & $\begin{array}{l}\text { Szabó, Réka; University of St Andrews, School of Biology } \\
\text { Ferrier, David; University of St. Andrews, The Gatty Marine Laboratory; }\end{array}$ \\
\hline \hline Keywords: & morphallaxis, lophotrochozoan, BrdU, Phospohistone H3, polychaete \\
\hline &
\end{tabular}

SCHOLARONE ${ }^{\mathrm{m}}$

Manuscripts 


\section{Cell proliferation dynamics in regeneration of the operculum head appendage in the annelid Pomatoceros lamarckii.}

Réka Szabó and David Ellard Keith Ferrier*.

The Scottish Oceans Institute, Gatty Marine Laboratory, University of St Andrews, East Sands, St Andrews, Fife, KY16 8LB, UK.

e-mail.

Réka Szabó, rs386@st-andrews.ac.uk

David E.K. Ferrier, dekf@st-andrews.ac.uk

Number of figures: 6

Abbreviated title: Pomatoceros operculum regeneration

*Correspondence to,

David E.K. Ferrier

e-mail: dekf@st-andrews.ac.uk

phone: 01334463480

The Scottish Oceans Institute, Gatty marine laboratory, University of St Andrews, East Sands, Fife, KY16 8LB, UK. 


\begin{abstract}
.
Regeneration of lost or damaged appendages is a widespread and ecologically important ability in the animal kingdom, and also of great significance to developing regenerative medicine. The operculum of serpulid polychaetes is one among the many diverse
\end{abstract} appendages found in the lophotrochozoan superphylum, a clade hitherto understudied with respect to the mechanisms of appendage regeneration. In this study, we establish the normal time course of opercular regeneration in the serpulid Pomatoceros lamarckii and describe cell proliferation patterns in the regenerating opercular filament. The P. lamarckii operculum regenerates through a rapid and consistent series of morphogenetic events. Based on 5-bromo-2'-deoxyuridine (BrdU) labelling and anti-phosphohistone H3 immunohistochemistry, opercular regeneration appears to be a mixture of an early morphallactic stage and a later phase characterised by widespread proliferative activity within the opercular filament. Tracking residual pigmentation suggests that the distal part of the stump gives rise to the most distal structures of the operculum via morphallactic remodelling, whereas more proximal structures are derived from the proximal stump. Our work underscores the diversity of regenerative strategies employed by animals and introduces $P$. lamarckii as an emerging model of appendage regeneration. 


\title{
Introduction.
}

\begin{abstract}
Appendages may be defined as outgrowths from an animal's body with their own axes of polarity distinct from the main body axis or axes. Appendages include the limbs of many vertebrates and arthropods, the various parapodia, palps and cirri of annelids, or the tentacles of cnidarians. Appendages contribute to the great morphological and ecological diversity of animals, and are often capable of some degree of regeneration when lost, thus providing useful and often amenable systems with which to investigate the mechanisms of regeneration.
\end{abstract}

To date, the mechanisms of appendage regeneration have been best studied in vertebrates, especially the limbs of urodele amphibians (reviewed in Brockes, '97; Nye et al., 2003; Stocum and Cameron, 2011). Among non-vertebrate deuterostomes, cell proliferation and morphogenesis have been described in arm regeneration in several echinoderms (Candia Carnevali et al., '95, '97; Moss et al., '98; Thorndyke et al., 2001; Biressi et al., 2010; Fan et al., 2011), and investigations into the molecular mechanisms of arm regeneration also exist (Thorndyke et al., 2001; Patruno et al., 2002; Bannister et al., 2005; Burns et al., 2011). However, whether the arms of echinoderms are true appendages (Hotchkiss, '98; Peterson et al., 2000), or instead are elements of the main body axis/axes is a contentious issue (Morris, 2012). It could be argued instead that the tube feet of echinoderms are the appendages in this phylum, in which case less is known about echinoderm appendage regeneration. 
The study of leg regeneration in arthropods is a significant contributor to classical developmental biology, with a wealth of $20^{\text {th }}$ century work concerning positional information, polarity, intercalation, and cell behaviour (Bohn, '70a, b; French et al., 1976; French, '78, '80, '82; Anderson and French, '85). More recently, arthropod leg regeneration has become the subject of renewed interest, including studies of molecular mechanisms (Nakamura et al., 2008; Bando et al., 2013; Shah et al., 2011; Mitten et al., 2012; Lee et al., 2013). In addition, imaginal discs in the holometabolous insect Drosophila melanogaster provide a study system that takes advantage of the sophisticated tools available in this species (see Bergantiños et al., 2010; Repiso et al., 2011; Worley et al., 2012 for reviews).

Vertebrates, echinoderms and arthropods span two of the three great bilaterian "superphyla": the Deuterostomia and the Ecdysozoa. Members of the third "superphylum", the Lophotrochozoa, also sport a great variety of appendages, many of which are capable of regeneration. These include the arms, siphons and sensory tentacles of molluscs (Lange, '20; Pekkarinen, '84; Chase and Kamil, '83; Bobkova et al., 2004), and the diverse anterior appendages of annelid worms (Bubel et al., '80, '85; Lindsay et al., 2007; Dualan and Williams, 2011;). Such appendages play an important ecological role. Sub-lethal predation on regenerative body parts such as bivalve siphons is a significant contributor to benthic productivity (reviewed in Lindsay, 2010). Also, regeneration may place a burden on the animal through, for example, the cost of re-growing the missing structure, impairment of activities such as feeding and reproduction during regeneration, and increasing predation risk (Maginnis, 2006). Ecophysiological aspects of appendage regeneration have been studied in a number of lophotrochozoan species (de Vlas, '85; Tomiyama and Ito, 2006; 
Berke et al., 2009; Nuñez et al., 2010, Dualan and Williams, 2011; Hoso, 2012). However, the mechanisms underlying lophotrochozoan appendage regeneration have thus far received much less attention.

\begin{abstract}
Planarians, which include some of the best regenerators in the Bilateria and undoubtedly the best-studied lophotrochozoan regeneration models, possess a pharynx that may be considered an appendage under our definition. However, relatively few studies address pharynx regeneration outside the context of a more general regeneration process (for a review of pharynx regeneration, see Kreshchenko, 2009).
\end{abstract}

Traditionally, regenerative processes have been classified in two broad categories defined by T. H. Morgan (Morgan, '01). Epimorphosis, or growth of undifferentiated tissue before differentiation of the new structure, can be contrasted with morphallaxis, or regeneration through remodelling of existing tissues without proliferation. Morgan emphasised that the distinction between the two is not necessarily sharp, and they may occur together in the same system. Recently, Agata et al. (2007) reiterated these points, and proposed distalisation and intercalation as a new unifying principle of regeneration. These authors argue that regardless of proliferation, the regeneration of lost structures in animals tends to proceed by forming the most distal part of the structure first, and intercalating the rest of the missing tissue between this and the remainder of the old structure. The diverse lophotrochozoan appendages represent an untapped resource to test the generality of this principle. 
The serpulid polychaete Pomatoceros lamarckii (Figure 1) provides an ideal system in which to investigate lophotrochozoan appendage regeneration. P. lamarckii is a common member of intertidal communities around the British Isles. This sessile suspension feeder lives in calcareous habitation tubes attached to hard substrata. It possesses two types of functionally important and highly regenerative head appendages. The radioles (tentacles) serve in food capture and respiration, while the operculum is a defensive structure that can close the tube when the animal withdraws, or autotomise as a sacrificial body part. Both structures regenerate rapidly when lost, and are commonly seen regenerating in wildcollected worms (personal observation), consistent with their importance to the animal. Preliminary descriptions of the histology of intact and regenerating opercular filaments in P. lamarckii have been previously provided (Hanson, 1949; Bubel et al., '80, '83, '85; Bubel and Thorp, '85). Here we establish the time-course of opercular regeneration using a large sample of worms, and describe cell proliferation patterns during regeneration. The $P$. lamarckii opercular filament regenerates quickly and consistently, unaffected by factors such as size, sex and non-life-threatening injuries. Opercular regeneration appears to involve morphallactic remodelling of existing tissue combined with subsequent extensive cell proliferation, especially in the epidermis. We provide preliminary evidence that $P$. lamarckii operculum regeneration operates via the type of 'distalisation-intercalation' process proposed by Agata et al (2007), and thus provide a description of a system for studying adult appendage regeneration in a lophotrochozoan invertebrate, with several features that should facilitate its establishment as a useful comparative model. 


\section{Materials and Methods.}

\section{Animal collection and husbandry}

Rocks with Pomatoceros lamarckii tubes were obtained from the intertidal rock pools at East Sands beach, St Andrews, Scotland, and kept in a circulating seawater aquarium system at ambient temperature. Adult worms were removed from their tubes by breaking the posterior end of the tube and gently pushing the worm out with blunt forceps. After detubing, animals were kept in filtered seawater (FSW) in an air-conditioned room at 16$17^{\circ} \mathrm{C}$. Worms for the cell proliferation experiments were housed in plastic Petri dishes (9 cm diameter) containing up to ten worms in 25-30 ml FSW changed every few days. Worms that were followed individually to record the time course of regeneration were kept in Nunclon four-well plates, one worm per well in $1 \mathrm{ml} \mathrm{FSW}$ changed daily. Opercular amputations were performed with a scalpel at the easy break point (Figure 1B).

\section{Time course of regeneration}

To establish the time course of regeneration, a sample of 100 worms was used. Immediately after detubing, each animal was photographed in left lateral view with a Nikon Coolpix 4500 camera mounted on a dissecting microscope. Worms were also sexed where possible (94/100), making use of the fact that detubing induces spawning in sexually mature individuals. The photographs were used to estimate the size of each worm as a proxy for age. Size was estimated using Image 1.46 and recorded as the distance (to the nearest 1/10 $\mathrm{mm}$ ) from the top of the folded-down collar to the last thoracic uncinus. These markers 
were chosen because they are relatively robust to the animal's movement and identifiable in imperfect photographs.

After amputation, each worm was observed daily for 14 days, and scored each day for the presence of seven morphogenetic landmarks as well as five components of pigmentation (Figure 2). In total, four animals were excluded from analysis: one died on day 4, one had a highly abnormal regenerate that was short and malformed from the early stages and failed to develop any pigmentation by the end of the observation period, another animal still had an open wound on its abdomen on day 14 , and in a fourth, the regenerate was hidden from view by the tentacles for most of the observation period.

\section{BrdU labelling and immunohistochemistry}

The S-phase marker 5-bromo-2'-deoxyuridine (BrdU) was used to assay cell proliferation during the first ten days of regeneration. BrdU was added to the water in the dishes of experimental animals for $48 \mathrm{~h}$ at a starting concentration of $1 \mathrm{mg} / \mathrm{ml}$. After the pulse, the worms were washed 3-5 times with clean FSW and checked for regeneration defects. Anterior portions were removed with a scalpel and fixed in 4\% paraformaldehyde (PFA) in $1 \mathrm{x}$ phosphate buffered saline (PBS) overnight at $4^{\circ} \mathrm{C}$. Fixed heads were washed three times in PBS before staining. Whole-mount specimens were stained as is, with head and thoracic tissue still present. To investigate proliferation in the interior of the opercular filament, some fixed regenerates were dissected from the head and cut into portions with a razorblade. 
The procedure for BrdU immunohistochemistry was adapted from de Rosa et al. (2005), with the following changes: proteinase K (Sigma molecular grade solution) was used at $\sim 270 \mu \mathrm{g} / \mathrm{ml}$ for 15 minutes to permeabilise the specimens, and antibody incubations were performed overnight at $4^{\circ} \mathrm{C}$. Specimens were stained using the 3,3'-diaminobenzidine (DAB; Sigma) substrate according to the manufacturer's instructions, rinsed four times to stop the reaction, and photographed under a dissecting microscope.

\section{Phosphohistone H3 immunohistochemistry}

As a second marker, we used the mitosis-specific phosphorylation of histone H3. Specimens for this experiment were decapitated as above, fixed for $30 \mathrm{~min}$ at room temperature, washed, and dissected to remove non-opercular tissue. The regenerates were permeabilised with a one-hour incubation in PBS with 2\% Triton X-100. For all other steps, PBS with 0.1\% Tween-20 (PBT) was used as a buffer. Permeabilisation was followed by three PBT washes, then incubation in block-PBT (PBT with 5\% sheep serum) for two hours. After this, a 1:500 dilution of rabbit polyclonal antibody against histone H3 phosphorylated on Ser28 (Millipore) was added for an overnight incubation at $4^{\circ} \mathrm{C}$. The primary antibody was removed with four PBT washes of at least 15 minutes each. Secondary incubation and staining was done with the Vectastain Elite ABC kit (Vector Laboratories). The antibody incubation was performed overnight at $4^{\circ} \mathrm{C}$, followed by four 15 -minute washes and incubation with the $\mathrm{ABC}$ reagent from the kit according to the manufacturer's instructions. The specimens were then washed again and stained with DAB as in the BrdU experiments. Stained and washed specimens were dehydrated through an ethanol series, mounted in 60\% glycerol, and photographed with a QImaging Retiga 2000R camera mounted on a Leica 
microscope equipped with Nomarski optics, using the QCapture Suite ${ }^{\mathrm{TM}}$ (version 2.9.3) or ImagePro® Insight version 8.

\section{Results}

The mature opercular filament

The anatomy of P. lamarckii and the opercular filament is shown in Figure 1. The cup- or funnel-shaped operculum sits on top of a stout peduncle. In the mature structure, the two regions are separated by a prominent groove. The peduncle has a triangular cross-section, more pronounced distally. An autotomy plane, called the easy break point, is situated partway down the peduncle (Figure 1B, dashed line); all experimental amputations in this study were carried out at or very near this point. The cup is closed distally by a flat or concave opercular plate. The plate bears a spine with two large dorsal prongs and a smaller ventral one; these are often eroded in wild worms or broken off during detubing. In $P$. lamarckii, the opercular plate and spine are calcified (Bubel et al., '83). The mature opercular filament is strikingly pigmented with a pattern of alternating white and dark bands. Pigmentation patterns display considerable individual variation, but several features, including a prominent dark band immediately distal to the easy break point, are generally recognisable (Figure 1B).

Time course of opercular regeneration 
Upon amputation at the easy break point, regeneration begins rapidly. Immediately after amputation, the end of the stump contracts so that there is virtually no blood loss from the large opercular blood vessel. The first sign of regeneration is the elongation of the stump and the emergence of the future prongs of the opercular spine from the tips of the triangular amputation surface. By one day post-operation (dpo), a small swelling is usually present around the middle of the stump (Figure 2B). This swelling subsequently enlarges (Figure 2C), develops a rim distinct from the base of the spine (Figure 2D), and becomes cup-shaped with an expanding distal plate (Figure 2E). Calcification (Figure 2F) normally becomes visible at the base of the spine soon after rim formation, often before there is a clear cup or plate. At 3 dpo, nearly all opercula are visibly calcifying. Once a cup is present, the groove between it and the peduncle begins to form. It first appears as a narrow line marking the previously smooth peduncle-operculum boundary (Figure 2G). Shortly after groove formation, wing buds develop on either side of the peduncle just below the groove (Figure 2H). After this point, no new anatomical structures appear, but existing structures such as wings continue to grow, and pigmentation is added.

The timing of these morphogenetic landmarks is largely consistent between individuals, with most animals reaching a given landmark within a day of each other. Although there is slight variation in their absolute timing, the order of the landmarks appears fixed, such that no regenerate develops a groove before it has a well-differentiated cup, etc. (data not shown). The relative timing of calcification and cup formation do vary slightly, but all animals in our sample began calcifying before the appearance of a groove (data not shown). 
New pigmentation does not develop until all of the morphogenetic landmarks described above have appeared. Usually, the first visible pigmentation is dispersed red or brown dots on the cup. Other elements of pigmentation that were scored in this study are the dark banding on the cup, the proximal and distal dark pigment bands of the peduncle, and the white banding on the peduncle and/or cup (Figure 1B; Figure 2J, drawings in Figure 2K). These appear much less constrained than the morphogenetic landmarks; both absolute timing and the order in which they appear is more variable (Figure $2 \mathrm{~K}$, data not shown), particularly for the dark bands in various locations.

To investigate potential factors influencing regeneration, we recorded the sex and approximate size of each animal, as well as any non-opercular injuries they sustained during detubing. Apart from extreme cases where injuries caused serious illness in the worm, these factors do not appreciably change the time course of regeneration. Comparative plots for sex and size are shown in Figure 3.

Cell proliferation patterns

We first used the S-phase marker BrdU to assay cell proliferation in regenerating opercula. Live animals were exposed to BrdU for $48 \mathrm{~h}$ before being fixed and stained. During the earliest stages of regeneration, relatively little proliferation is detected (Figure 4A; 40 animals labelled at 0-2 dpo). However, the number of labelled cells in the epidermis increases dramatically during rim and cup morphogenesis (Figure 4B), and remains at high levels until morphogenesis is virtually complete (Figure 4C-D). Of 125 animals labelled in 1- 
3 dpo to 5-7 dpo pulses, 65 showed staining in both the cup wall and peduncle, and a further 31 displayed partial staining patterns (e.g. cup only). In late-stage regenerates (8-10 dpo), staining is often concentrated in the developing wings and the associated lateral ridges along the distal peduncle (Figure 4E-F; 21/24 animals showed this pattern compared to $9 / 37$ for 4-6 dpo). During peak proliferation, epidermal staining is present from the base of the peduncle to the wall of the cup, with no obvious regionalisation within this area. Notably, no similar proliferation increase was observed outside the regenerate, although the whole-mount BrdU experiments included substantial amounts of anterior tissue. The (presumptive) rim, plate and spine remain unstained throughout regeneration. Control heads from mid-regeneration stages (4-6 dpo) that received no BrdU treatment or no primary antibody $(\mathrm{n}=10$ each) display only a faint, even background throughout the opercular filament (Supplementary Figure).

Since the opercular filament is a large, opaque structure, we cut portions of tissue from opercula and peduncles to be able to examine proliferation patterns inside the structure. Proliferation in the connective tissue, muscle and the wall of the blood vessel appears to lag behind the epidermis, remaining low or undetectable until a well-developed cup is present (Figure 4G-H; 5/6 0-2 dpo specimens with a cup had staining in epidermis but not mesodermal tissues; 7/8 labelled at 1-3 dpo show strong mesodermal staining). From cup stage, labelled cells are abundant in the connective tissue and muscle within the peduncle (5/7 peduncle pieces from 5 animals at 4-6 dpo), as well as the wall of the blood vessel (Figure $4 \mathrm{H}-\mathrm{I}, \mathrm{K}$ ). Interestingly, the connective tissue inside the cup is always unstained, in sharp contrast to the blood vessel, which is densely stained during mid-regeneration 
(Figure 4K; 5/5 cups). Proliferation in the peduncle connective tissue, muscle and the blood vessel wall seems to drop off in the 8-10 dpo sample, even though epidermal proliferation in the cup wall may still be high at this time (Figure 4J, L; 7/7 cups and 9/12 peduncle pieces from 7 animals).

In addition to BrdU labelling, we employed antibodies against the mitotic marker phosphohistone $\mathrm{H} 3$ (PH3) to get finer time resolution than the requisite long BrdU pulses. In accordance with its 'snapshot' nature, $\mathrm{PH} 3$ immunohistochemistry detects far fewer cells than do the BrdU experiments. Proliferation in connective tissue and muscle is generally too low to be detectable with this method, and even the blood vessel inside the cup is largely negative. The distribution of epidermal staining is consistent with our observations with BrdU. From cup formation onwards, PH3-positive cells are present throughout the cup wall and the entire length of the peduncle (Figure 5), confirming the lack of regionalisation seen with BrdU (>100 specimens tested). PH3 staining also allowed us to examine stages earlier than 2 dpo. At 8 hpo, no proliferation is seen with this marker $(n=12)$, but a few cells are stained in 1 dpo regenerates with small swellings ( $>30$ animals tested)..

\footnotetext{
Prompted by the lack of proliferation in the plate region, we conducted a preliminary investigation into the origin of the distal structures of the operculum using residual stump pigmentation as a simple lineage-tracing tool. White pigmentation, when present, is often patchy, allowing fine-scale tracking of its fate. Our observations thus far show recognisable patterns of stump pigmentation carried over onto the forming distal structures, but not to any structure proximal to the opercular rim. An example time series is shown in Figure 6.
} 


\section{Discussion}

The morphogenesis of the $P$. lamarckii operculum during regeneration follows a stereotyped sequence of events. Bubel and Thorp ('85) noted that the opercular filament regenerates without a blastema. Our observations agree with this assessment. At the amputation surface, the earliest sign of regeneration is the formation of prongs, without any sign of an undifferentiated growth preceding morphogenesis.

It should be noted that cup morphogenesis from a smooth swelling is a continuum, and furthermore, some of our morphogenetic landmarks are logically dependent on each other (e.g. it would be difficult to imagine cup formation without a swelling). Nevertheless, with the exception of calcification, landmarks that could occur in varying orders (e.g. rims and grooves, or prongs and cups) in fact develop in a consistent order, indicating a tightly regulated developmental program. Neither this order nor the absolute timing of events appears affected by factors one might expect to influence regeneration, such as sex (Nachtrab et al., 2011) or size, which we used as a rough proxy for age (Tartakovskaya et al., 2003; Somorjai et al., 2012; Seifert and Voss, 2013). One caveat to this size/age conclusion is that, although they covered a range of sizes (thorax length 1.3-3.2 mm), nearly all of our animals (91/96 in the final sample) were reproductively active, and therefore certainly could be considered adults. Thus, while age does not seem relevant to the study of adult regeneration in $P$. lamarckii, age-related differences in regeneration between adults and juveniles cannot be excluded. 
Opercular pigmentation develops after morphogenesis is essentially complete, and shows more variation in timing and order than does the development of anatomical landmarks. Pigmentation also displays considerable variation in mature opercular filaments. The ecological aspects of operculum regeneration are beyond the scope of this study, but we might hypothesise that the presence of a complete opercular filament with a differentiated cup, plate and peduncle is more important for its defensive function than the presence or precise pattern of pigmentation. This may explain the greater variability and longer time scales seen in the regeneration of pigmentation compared to morphology.

\section{Cell proliferation and morphallaxis}

It is clear from our results that opercular morphogenesis in P. lamarckii begins without extensive proliferation. Therefore, early opercular regeneration fits Morgan's original definition of morphallaxis as the transformation of a part "directly into a new organism or part of an organism without proliferation at the cut-surfaces" (Morgan, '01, p. 23). Since Morgan's time, the definition of morphallaxis has broadened to include any reorganisation of pre-existing tissue during regeneration. Early opercular regeneration appears to be a largely morphallactic process in both senses. Later, cell proliferation does occur throughout most of the opercular filament, including the base of the peduncle, which is approximately $0.5 \mathrm{~mm}$ proximal to the level of amputation. A similar spread of proliferative cells beyond the level of amputation was observed in regenerating heads of the oligochaete annelid Pristina leidyi (Zattara and Bely, 2011), the arms of the crinoid Antedon mediterranea 
(Candia Carnevali et al., '97) and the regenerating tail of the cephalochordate Branchiostoma lanceolatum (Somorjai et al., 2012), all of which regenerate via a blastema. In contrast, in regenerating heads of the polychaete Dorvillea bermudensis (Paulus and Müller, 2004) and arms of the brittle star Ophiotrix fragilis (Thorndyke et al., 2001), proliferation is almost exclusive to the blastema, underscoring the diversity of the regenerative strategies employed by animals. Cell proliferation far away from the regenerate itself has been described, or at least suggested, for some annelids in the context of segment regeneration (Sugio et al., 2012). In planarians, proliferation does not occur in the regeneration blastema itself. Upon amputation, a proliferative response is seen not only near the blastema but also in body regions far from the amputation site (Saló and Baguñà, '84), and neoblasts appear capable of migrating long distances in order to contribute to regeneration (Reddien and Sánchez Alvarado, 2004). In particular, the planarian pharynx, which might be described as an appendage, appears to rely on the proliferation and migration of neoblasts anterior to the pharynx for regeneration (Ito et al., 2001).

Our observations with phosphohistone H3 (Figure 5) confirm that the global distribution of BrdU-positive cells within the opercular filament accurately represents the distribution of in situ proliferation rather than that of the descendants of a more localised proliferation zone. It will be interesting to investigate the mechanisms that trigger a proliferative response at a distance from the wound. Does the operculum possess a signalling centre that can convey signals to more proximal portions of the stump? For example, Bubel et al. ('85) observed that the cells of the future opercular rim are morphologically distinct from the rest of the epithelium already at the swelling stage. Could early-differentiating structures 
such as these serve as a source of long-range signals? It is also tempting to speculate about a role for the opercular nerves. Innervation is known to control the proliferative response in vertebrate limb regeneration (Stocum, 2011; Kumar and Brockes, 2012), and injury to one of the three opercular nerves can trigger the development of a new operculum in another serpulid (Schochet, '73).

\begin{abstract}
BrdU labelling indicates a time delay between proliferation in the epidermis and in the internal tissues, including the connective tissue, muscle and blood vessel wall. Similar delays have been observed in segment regeneration in other annelids (Marilley and Thouveny, '78; Yoshida-Noro and Tochinai, 2010); however, in both of the cited cases, proliferation in the internal tissues preceded that in the epidermis in contrast to the $P$. lamarckii situation.
\end{abstract}

\begin{abstract}
A striking observation from both of our markers is that there appears to be no proliferation at any stage of regeneration in the opercular rim, plate and spine. Bubel et al. ('85) remarked on the lack of mitotic figures in the same regions. Thus, three independent approaches confirm the lack of proliferation in the distal-most portion of an opercular regenerate. Since BrdU exposure affected whole animals, the lack of BrdU staining in this region also excludes migration of cells that proliferated elsewhere, although it does not exclude the participation of cells that went through S-phase before amputation (Nishimura et al., 2011). This strongly suggests that these distal structures are of morphallactic origin, and based on our BrdU results, the same is likely true of the connective tissue inside the cup.
\end{abstract}


Given the outcome of the cell proliferation assays, we hypothesised that the rim, plate and spine derived from the tissues of the stump through morphallactic remodelling. As a preliminary test of this hypothesis, we followed the fate of the pigmentation found on a significant fraction of proximal peduncles. White pigmentation is often patchy, forming unique patterns that can be tracked throughout regeneration. Our observations (exemplified by Figure 6) indicate that all of the residual white pigmentation is pushed to the distal portion of the regenerate and stretched, mostly occupying the spine and plate by the time a cup is formed. If the amputation did not occur precisely at the easy break point, remnants of the proximal pigment band can be present at the tip of the stump (Figure 6A), but these appear to be discarded early in regeneration (Figure 6B). Thus, we provisionally conclude that the distal half of the peduncle stump is the exclusive source of the new plate and spine region, while the cup wall and peduncle develop through the proliferation of the proximal stump. In the future, lineage-tracing approaches such as Dil labelling could offer a more rigorous test of this hypothesis.

\section{Opercular regeneration in P. lamarckii appears to be in good agreement with Agata et al.'s} (2007) recently proposed unifying principle of regeneration. These authors observed that regenerating structures in animals tend to form by distalisation and intercalation regardless of the mode of regeneration (epimorphic, morphallactic or mixed). Consistent with this model, in P. lamarckii the distal-most structure, i.e. the end of the spine, forms early, while more proximal structures, i.e. the cup and peduncle, later intercalate between these and the base of the old peduncle. Remarkably, some distal structures also appear to 
form directly from pre-existing tissue, while the peduncle and the cup wall are derived largely from the subsequent phase of proliferation. However, distalisation and intercalation is by no means established as a universal principle. For example, Roensch et al. (2013) recently argued, based on Hox protein expression patterns and cell transplantation experiments, that regenerating salamander limbs establish their positional identities in a proximodistal sequence. Until reliable molecular markers of proximal and distal identities are established for the P. lamarckii opercular filament, the possibility of alternative scenarios cannot be conclusively excluded.

Lophotrochozoans represent a diverse and largely unexploited resource for studying the mechanisms of appendage regeneration in metazoans. As a common and easily maintained animal with rapidly regenerating head appendages, the serpulid polychaete $P$. lamarckii offers a tractable system for such research. In this study, we established a time course and cell proliferation dynamics of the regeneration of the operculum, a unique appendage of serpulids. Based on a sample of 100 worms, opercular regeneration in P. lamarckii follows a consistent series of morphogenetic events whose timing is unaffected by factors such as sex and size. Cell proliferation in the regenerating opercular filament extends to the base of the peduncle well below the level of amputation, but not to areas outside the opercular filament. It will be interesting to determine the nature of the signals that regulate the boundary of the proliferative response. Also, P. lamarckii operculum regeneration appears to be an unusual mixture of morphallactic and epimorphic elements, with the opercular spine, plate and rim forming without detectable proliferation, and the cup, peduncle and wings differentiating from an actively proliferating region. The fate of residual stump 
pigmentation suggests that tissue distal to the easy break point is not incorporated into the regenerate, morphallactic remodelling happens in the distal half of the stump, and the peduncle and cup intercalate behind this remodelling zone. Thus, opercular regeneration appears to be an example of Agata et al.'s (2007) distalisation-intercalation model.

\section{Acknowledgments}

The authors would like to thank the members of the Ferrier and Somorjai labs for discussions, and Sarah Miles for preliminary experiments on Pomatoceros regeneration. RS is supported by a Carnegie Scholarship. 


\title{
Literature Cited
}

Agata K, Saito Y, Nakajima E. 2007. Unifying principles of regeneration I: Epimorphosis versus morphallaxis. Dev Growth Differ 49:73-78.

\begin{abstract}
Anderson H, French V. 1985. Cell division during intercalary regeneration in the cockroach leg. J Embryol Exp Morphol 90:57-78.
\end{abstract}

\begin{abstract}
Bando T, Ishimaru Y, Kida T, Hamada Y, Matsuoka Y, Nakamura T, Ohuchi H, Noji S, Mito T. 2013. Analysis of RNA-Seq data reveals involvement of JAK/STAT signalling during leg regeneration in the cricket Gryllus bimaculatus. Development 140:959-964.
\end{abstract}

\begin{abstract}
Bannister R, McGonnell IM, Graham A, Thorndyke MC, Beesley PW. 2005. Afuni, a novel transforming growth factor- $\beta$ gene is involved in arm regeneration by the brittle star Amphiura filiformis. Dev Genes Evol 215:393-401.
\end{abstract}

\begin{abstract}
Bergantiños C, Vilana X, Corominas M, Serras F. 2010. Imaginal discs: Renaissance of a model for regenerative biology. BioEssays 32:207-217.
\end{abstract}


Berke SK, Cruz V, Osman RW. 2009. Sublethal Predation and Regeneration in Two Onuphid Polychaetes: Patterns and Implications. Biol Bull 217:242-252.

Biressi ACM, Zou T, Dupont S, Dahlberg C, Benedetto CD, Bonasoro F, Thorndyke M, Carnevali MDC. 2010. Wound healing and arm regeneration in Ophioderma longicaudum and Amphiura filiformis (Ophiuroidea, Echinodermata): comparative morphogenesis and histogenesis. Zoomorphology 129:1-19.

\begin{abstract}
Bobkova MV, Tartakovskaya OS, Borissenko SL, Zhukov VV, Meyer-Rochow VB. 2004. Restoration of morphological and functional integrity in the regenerating eye of the giant African land snail Achatina fulica. Acta Zoologica 85:1-14.
\end{abstract}

\begin{abstract}
Bohn H. 1970a. Interkalare Regeneration und segmentale Gradienten bei den Extremitäten von Leucophaea-Larven (Blattaria). Wilhelm Roux' Arch Entwickl Mech Org 165:303-341.
\end{abstract}

Bohn H. 1970b. Interkalare Regeneration und segmentale Gradienten bei den Extremitäten von Leucophaea-Larven (Blattaria): II. Coxa und Tarsus. Dev Biol 23:355-379. 
Brockes JP. 1997. Amphibian Limb Regeneration: Rebuilding a Complex Structure. Science 276:8187.

Bubel A, Thorp CH, Moore MN (1980) An histological, histochemical and ultrastructural study of the operculum of the serpulid Pomatoceros triqueter L. with particular reference to the formation of the calcareous opercular plate during opercular regeneration. In Oxley TA, Becker G, Allsopp D (eds.) Biodeterioration: The Proceedings of the Fourth International Biodeterioration Symposium. London: Biodeterioration Society, pp. 275-290

Bubel A, Stephens RM, Fenn RH, Fieth P. 1983. An electron microscope, X-ray diffraction and amino acid analysis study of the opercular filament cuticle, calcareous opercular plate and habitation tube of Pomatoceros lamarckii Quatrefages (Polychaeta: Serpulidae). Comp Biochem Phys B 74:837-850.

Bubel A, Thorp CH. 1985. Tissue abscission and wound healing in the operculum of Pomatoceros lamarckii Quatrefages (Polychaeta: Serpulidae). J Zool 1:95-143.

\footnotetext{
Burns G, Ortega-Martinez O, Thorndyke MC, Peck LS, Dupont S, Clark MS. 2011. Dynamic gene expression profiles during arm regeneration in the brittle star Amphiura filiformis. J Exp Mar Biol Ecol 407:315-322.
}

John Wiley \& Sons 
Candia Carnevali MD, Bonasoro F, Biale A. 1997. Pattern of bromodeoxyuridine incorporation in the advanced stages of arm regeneration in the feather star Antedon mediterranea. Cell Tissue Res 289:363-374.

Candia Carnevali MD, Bonasoro F, Lucca E, Thorndyke MC. 1995. Pattern of cell proliferation in the early stages of arm regeneration in the feather star Antedon mediterranea. J Exp Zool 272:464-474.

Chase R, Kamil R. 1983. Morphology and odor sensitivity of regenerated snail tentacles. J Neurobiol $14: 43-50$.

de Rosa R, Prud'homme B, Balavoine G. 2005. caudal and even-skipped in the annelid Platynereis dumerilii and the ancestry of posterior growth. Evolution \& Development 7:574587.

De Vlas J. 1985. Secondary production by siphon regeneration in a tidal flat population of Macoma balthica. Neth J Sea Res 19:147-164.

Dualan IV, Williams JD. 2011. Palp growth, regeneration, and longevity of the obligate hermit crab symbiont Dipolydora commensalis (Annelida: Spionidae). Invertebr Biol 130:264-276. 
Fan T, Fan X, Du Y, Sun W, Zhang S, Li J. 2011. Patterns and cellular mechanisms of arm regeneration in adult starfish Asterias rollestoni bell. J Ocean Univ China 10:255-262.

French V, Bryant PJ, Bryant SV. 1976. Pattern regulation in epimorphic fields. Science 193:969-981.

French V. 1978. Intercalary regeneration around the circumference of the cockroach leg. J Embryol Exp Morphol 47:53-84.

French V. 1980. Positional information around the segments of the cockroach leg. J Embryol Exp Morphol 59:281-313.

French V. 1982. Leg Regeneration in Insects: Cell Interactions and Lineage. Amer Zool 22:79-90.

Hanson J. 1949. Observations on the Branchial Crown of the Serpulidae (Annelida, Polychaeta). Q J Microsc Sci s3-90:221-233.

John Wiley \& Sons 
Hoso M. 2012. Cost of autotomy drives ontogenetic switching of anti-predator mechanisms under developmental constraints in a land snail. Proc R Soc B 279:4811-4816.

Hotchkiss FHC. 1998. A "Rays-as-Appendages" Model for the Origin of Pentamerism in Echinoderms. Paleobiology 24:200-214.

Ito H, Saito Y, Watanabe K, Orii H. 2001. Epimorphic regeneration of the distal part of the planarian pharynx. Dev Genes Evol 211:2-9.

Kreshchenko ND. 2009. Pharynx regeneration in planarians. Russ J Dev Biol 40:1-13.

Kumar A, Brockes JP. 2012. Nerve dependence in tissue, organ, and appendage regeneration. Trends Neurosci 35:691-699.

Lange MM. 1920. On the regeneration and finer structure of the arms of the cephalopods. J Exp Zool $31: 1-57$. 
Lee AK, Sze CC, Kim ER, Suzuki Y. 2013. Developmental coupling of larval and adult stages in a complex life cycle: insights from limb regeneration in the flour beetle, Tribolium castaneum. EvoDevo 4:1-17.

Lindsay SM, Jackson JL, He SQ. 2007. Anterior regeneration in the spionid polychaetes Dipolydora quadrilobata and Pygospio elegans. Mar Biol 150:1161-1172.

Lindsay SM. 2010. Frequency of Injury and the Ecology of Regeneration in Marine Benthic Invertebrates. Integr Comp Biol 50:479-493.

\begin{abstract}
Maginnis TL. 2006. The costs of autotomy and regeneration in animals: a review and framework for future research. Behav Ecol 17:857-872.
\end{abstract}

\begin{abstract}
Marilley M, Thouveny Y. 1978. DNA synthesis during the first stages of anterior regeneration in the polychaete annelid Owenia fusiformis (dedifferentiation and early phases of differentiation ). J Embryol Exp Morphol 44:81-92.
\end{abstract}

John Wiley \& Sons 
Mitten EK, Jing D, Suzuki Y. 2012. Matrix metalloproteinases (MMPs) are required for wound closure and healing during larval leg regeneration in the flour beetle, Tribolium castaneum. Insect Biochem Mol Biol 42:854-864.

Morgan TH. 1901. Regeneration. New York: Macmillan.

Morris VB. 2012. Early development of coelomic structures in an echinoderm larva and a similarity with coelomic structures in a chordate embryo. Dev Genes Evol 222:313-323.

\begin{abstract}
Moss C, Hunter AJ, Thorndyke MC. 1998. Patterns of bromodeoxyuridine incorporation and neuropeptide immunoreactivity during arm regeneration in the starfish Asterias rubens. Phil Trans R Soc Lond B 353:421-436.
\end{abstract}

\author{
Nachtrab G, Czerwinski M, Poss KD. 2011. Sexually Dimorphic Fin Regeneration in Zebrafish \\ Controlled by Androgen/GSK3 Signaling. Current Biology 21:1912-1917
}

\begin{abstract}
Nakamura T, Mito T, Miyawaki K, Ohuchi H, Noji S. 2008. EGFR signaling is required for reestablishing the proximodistal axis during distal leg regeneration in the cricket Gryllus bimaculatus nymph. Dev Biol 319:46-55.
\end{abstract}




\begin{abstract}
Nishimura K, Inoue T, Yoshimoto K, Taniguchi T, Kitamura Y, Agata K. 2011. Regeneration of dopaminergic neurons after 6-hydroxydopamine-induced lesion in planarian brain. J Neurochem
\end{abstract} 119:1217-1231.

\begin{abstract}
Nuñez JD, Ocampo EH, Chiaradia NM, Morsan E, Cledón M. 2013. The effect of temperature on the inhalant siphon regeneration of Amiantis purpurata (Lamarck, 1818) (Bivalvia; Veneridae). Mar Biol Res 9:189-197.
\end{abstract}

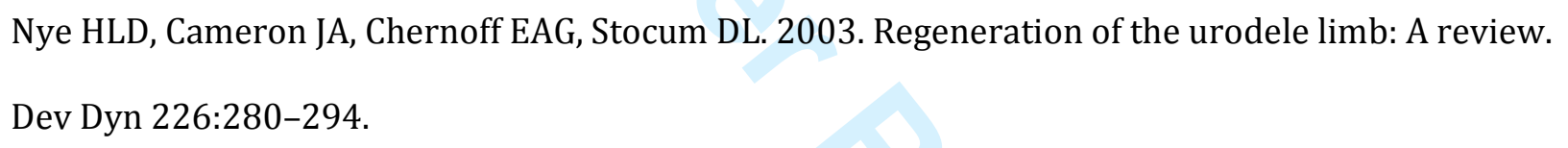

Patruno M, Smertenko A, Carnevali MDC, Bonasoro F, Beesley PW, Thorndyke MC. 2002. Expression of transforming growth factor $\beta$-like molecules in normal and regenerating arms of the crinoid Antedon mediterranea: immunocytochemical and biochemical evidence. Proc R Soc Lond B 269:1741-1747.

\footnotetext{
Paulus T, Müller MCM. 2004. Cell proliferation dynamics and morphological differentiation during regeneration in Dorvillea bermudensis (Polychaeta, Dorvilleidae). J Morphol 267:393-403.
} 
Pekkarinen M. 1984. Regeneration of the inhalant siphon and siphonal sense organs of brackishwater (Baltic Sea) Macoma balthica (Lamellibranchiata, Tellinacea). Ann Zool Fenn 21:29-40.

Peterson KJ, Arenas-Mena C, Davidson EH. 2000. The A/P axis in echinoderm ontogeny and evolution: evidence from fossils and molecules. Evol Dev 2:93-101.

Reddien PW, Sánchez Alvarado A. 2004. Fundamentals of Planarian Regeneration. Annu Rev Cell Dev Biol 20:725-757.

Repiso A, Bergantiños C, Corominas M, Serras F. 2011. Tissue repair and regeneration in Drosophila imaginal discs. Dev Growth Differ 53:177-185.

Roensch K, Tazaki A, Chara O, Tanaka EM. 2013. Progressive specification rather than intercalation of segments during limb regeneration. Science 342:1375-1379.

Saló E, Baguñà J. 1984. Regeneration and pattern formation in planarians I. The pattern of mitosis in anterior and posterior regeneration in Dugesia (G) tigrina, and a new proposal for blastema formation. J Embryol Exp Morphol 83:63-80. 
Schochet J. 1973. Opercular regulation in the polychaete Hydroides dianthus (Verrill, 1873) II. Control of opercular regulation. J Exp Zool 184:259-279.

Seifert AW, Voss SR. 2013. Revisiting the relationship between regenerative ability and aging. BMC Biol 11:2.

Shah MV, Namigai EKO, Suzuki Y. 2011. The role of canonical Wnt signaling in leg regeneration and metamorphosis in the red flour beetle Tribolium castaneum. Mech Develop 128:342-358.

Somorjai IML, Somorjai RL, Garcia-Fernàndez J, Escrivà H. 2012. Vertebrate-like regeneration in the invertebrate chordate amphioxus. PNAS 109:517-522.

Stocum DL, Cameron JA. 2011. Looking proximally and distally: 100 years of limb regeneration and beyond. Dev Dyn 240:943-968.

Stocum DL. 2011. The role of peripheral nerves in urodele limb regeneration. Eur J Neurosci 34:908-916. 
Sugio M, Yoshida-Noro C, Ozawa K, Tochinai S. 2012. Stem cells in asexual reproduction of Enchytraeus japonensis (Oligochaeta, Annelid): Proliferation and migration of neoblasts. Dev Growth Differ 54:439-450.

Tartakovskaya OS, Borisenko SL, Zhukov VV. 2003. Role of the Age Factor in Eye Regeneration in the Gastropod Achatina fulica. Biol Bull 30:228-235.

Thorndyke MC, Chen W-C, Beesley PW, Patruno M. 2001. Molecular approach to echinoderm regeneration. Microsc Res Tech 55:474-485.

Tomiyama T, Ito K. 2006. Regeneration of lost siphon tissues in the tellinacean bivalve Nuttallia olivacea. J Exp Mar Biol Ecol 335:104-113.

Worley MI, Setiawan L, Hariharan IK. 2012. Regeneration and Transdetermination in Drosophila Imaginal Discs. Annu Rev Genet 46:289-310.

Yoshida-Noro C, Tochinai S. 2010. Stem cell system in asexual and sexual reproduction of Enchytraeus japonensis (Oligochaeta, Annelida). Dev Growth Differ 52:43-55. 
Zattara EE, Bely AE. 2011. Evolution of a novel developmental trajectory: fission is distinct from regeneration in the annelid Pristina leidyi. Evol Dev 13:80-95. 


\section{Figure legends}

Figure 1. General anatomy of Pomatoceros lamarckii and the operculum. A. Adult P. lamarckii removed from its tube. Right lateral view, anterior to the top. B. Close-up of the opercular filament from $\mathbf{A}$. Abbreviations are as follows: $\mathbf{p p b}=$ proximal pigment band, $\mathbf{d p b}=$ distal pigment band, $\mathbf{g}=$ groove between the peduncle and the cup (= operculum proper), $\mathbf{w}=$ wing, $\mathbf{p l}=$ opercular plate, $\mathbf{s p}$ $=$ spine. The dashed line marks the easy break point, the site of autotomy and experimental amputation throughout this study. Scale bars are approximately $1 \mathrm{~mm}$.

\section{Figure 2. The time course of opercular regeneration. A-J - stages of regeneration. All} photographs are in dorsal view except F, G and J (left lateral). Scale bars are approximately $0.5 \mathrm{~mm}$. A. Peduncle stump shortly after amputation at the easy break point. The triangular end of the stump has contracted to close the wound. B. Initiation/early swelling (specimen pictured is 1 day postoperation [dpo]). The stump has elongated, and the prongs (arrow) of the future spine have begun to form from the corners of the amputation surface. Most specimens are also beginning to show a swelling (arrowhead) by 1 dpo. C. Large swelling (specimen is 2 dpo). D. Rim formation (specimen is $2 \mathrm{dpo}$ ). Arrowhead marks the opercular rim differentiating from the base of the spine. E. Cup formation (specimen is $2 \mathrm{dpo}$ ). The opercular plate expands and the swelling becomes cup-shaped. F. Calcification (specimen is $2 \mathrm{dpo}$ ). G. Groove formation (specimen is $5 \mathrm{dpo}$ ). The groove separating the peduncle from the cup is first visible as a narrow line (arrowhead) where previously there was a smooth boundary. H. Wing bud formation (specimen is $6 \mathrm{dpo}$ ). The lateral wings begin to form as small triangular protrusions at the end of the peduncle. Inset shows close-up of boxed area, with arrowhead marking the wing bud. I. Dotted pigmentation on the cup (specimen is $6 \mathrm{dpo}$ ). Inset shows close-up of the boxed area. J. 14 dpo regenerate displaying all elements of the mature pigment pattern. K. Timing of regeneration stages in a sample of 96 worms. The boxes represent 
interquartile ranges (IQR), with a median line and whiskers extending to $1.5 \mathrm{IQR}$. Diagrammed morphogenetic stages under the boxes correspond to those pictured in B-H as indicated by the letters below the drawings. The appearance of pigmentation (I-J, Fig. 1B) is further subdivided to record appearances of the following components, sketched below each box: dotted pigmentation on cup, dark banding on cup, proximal peduncular pigment band, distal peduncular pigment band, white banding on cup and/or peduncle. The numbers under each stage represent the number of animals that reached that stage before the end of the 14-day observation period or before they aborted their first regenerate and restarted regeneration $(n=7)$.

Figure 3. Size and sex do not affect regeneration. Comparison of the time course of regeneration between A. females $(n=49)$ and males $(n=42)$, B. lower quartile of thorax length $(1.3-2.2 \mathrm{~mm}, n=$ 30) and upper quartile $(2.5-3.1 \mathrm{~mm}, n=27)$. Stages are the same as Fig. 2.

Figure 4. BrdU labelling of regenerating opercula. A-F whole heads, G-L portions of peduncles and cups. In G-L, the inset drawings indicate the approximate location of the cut surface imaged, and the asterisks mark the lumen of the opercular blood vessel. Scale bars are approximately $0.5 \mathrm{~mm}$ in B-F, K and L, and $0.2 \mathrm{~mm}$ in A and G-J. A. 0-2 dpo pulse, early swelling stage regenerate. B. 1-3 dpo pulse, slightly end-on view of rim stage regenerate. Note the unstained presumptive plate and spine region. The out of focus tip of the spine has non-specific staining. C. 2-4 dpo pulse. Inset shows magnification of the boxed area. D. 4-6 dpo pulse. Inset shows unstained plate of the same specimen. E. 8-10 dpo pulse. F. Right lateral view of the specimen in E, showing the heavily labelled lateral ridge and wing. Inset shows close-up of the right wing from the boxed area. G. Early cup stage specimen (pulse 0-2 dpo) cut just below the cup. Arrowheads mark the thickness of the 
epidermis. The gaps between the epidermis and internal tissues in $\mathrm{G}$ and $\mathrm{H}$ are regions where the epidermal and mesodermal tissues have become detached from each other during the staining procedure. H. Same cut in a regenerate with a well-developed cup (pulse 1-3 dpo). I. Mid-peduncle cut of a 4-6 dpo specimen. J. Mid-peduncle cut of an 8-10 dpo specimen; note greatly decreased density of staining compared to I. K. Oblique cut through the cup of a 4-6 dpo specimen. Note strongly stained blood vessel wall and lack of labelled cells in the cup mesenchyme. The fainter staining around the blood vessel is located in the far wall of the cup. L. Distal cup of an 8-10 dpo specimen. Note that the cut sliced through the opercular blood vessel, which forms a large blindended spiral inside the cup. Three portions of the lumen are exposed (asterisks), and the wall of the vessel is unstained.

\author{
Figure 5. Epidermal phosphohistone $\mathrm{H} 3$ staining is present throughout the opercular \\ filament. The squares in A indicate the approximate locations of regions shown in B-D. B. Dorsal \\ cup wall of a 3 dpo specimen. C. Peduncle of a different 3 dpo specimen. D. Base of the peduncle in a \\ 2 dpo specimen. Scale bars represent $100 \mu \mathrm{m}$.
}

\begin{abstract}
Figure 6. Possible morphallaxis in the operculum. A single regenerating operculum pictured in left lateral view A. immediately after amputation, with residual white and grey pigmentation, and at B. 1 dpo, C. 2 dpo, and D. 3 dpo. Note how the pattern of white pigmentation appears to remain intact but transform as the plate and spine form. The arrow and arrowhead each mark the same white spot across the panels. Scale bars are approximately $0.5 \mathrm{~mm}$.
\end{abstract}


Supplementary Figure. Control experiments for BrdU labelling. A. 4 dpo specimen without BrdU treatment, but subjected to the full staining protocol. B. BrdU-treated specimen (4-6 dpo) stained without primary antibody. All specimens in both samples ( $\mathrm{n}=10 \mathrm{each})$ appear the same with noticeable background colouration when stained for long enough, but no specific nuclear staining. Dorsal views; scale bars are approximately $0.5 \mathrm{~mm}$. 

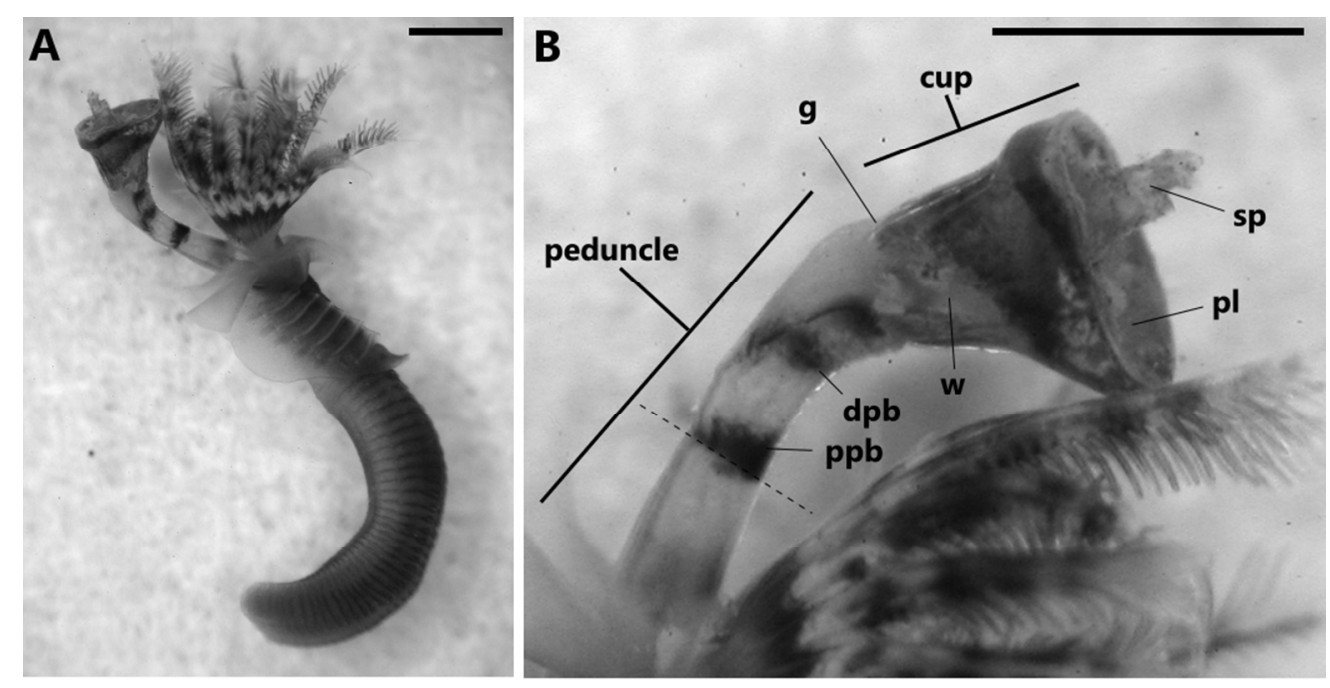

$368 \times 187 \mathrm{~mm}(72 \times 72$ DPI $)$

1
2
3
4
5
6
7
8
9
10
11
12
13
14
15
16
17
18
19
20
21
22
23
24
25
26
27
28
29
30
31
32
33
34
35
36
37
38
39
40
41
42
43
44
45
46
47
48
49
50
51
52
53
54
55
56
57
58
59
60

36

37

39

40

41

42

44

45

46

47

48

49

51

52

53

54

55

56 

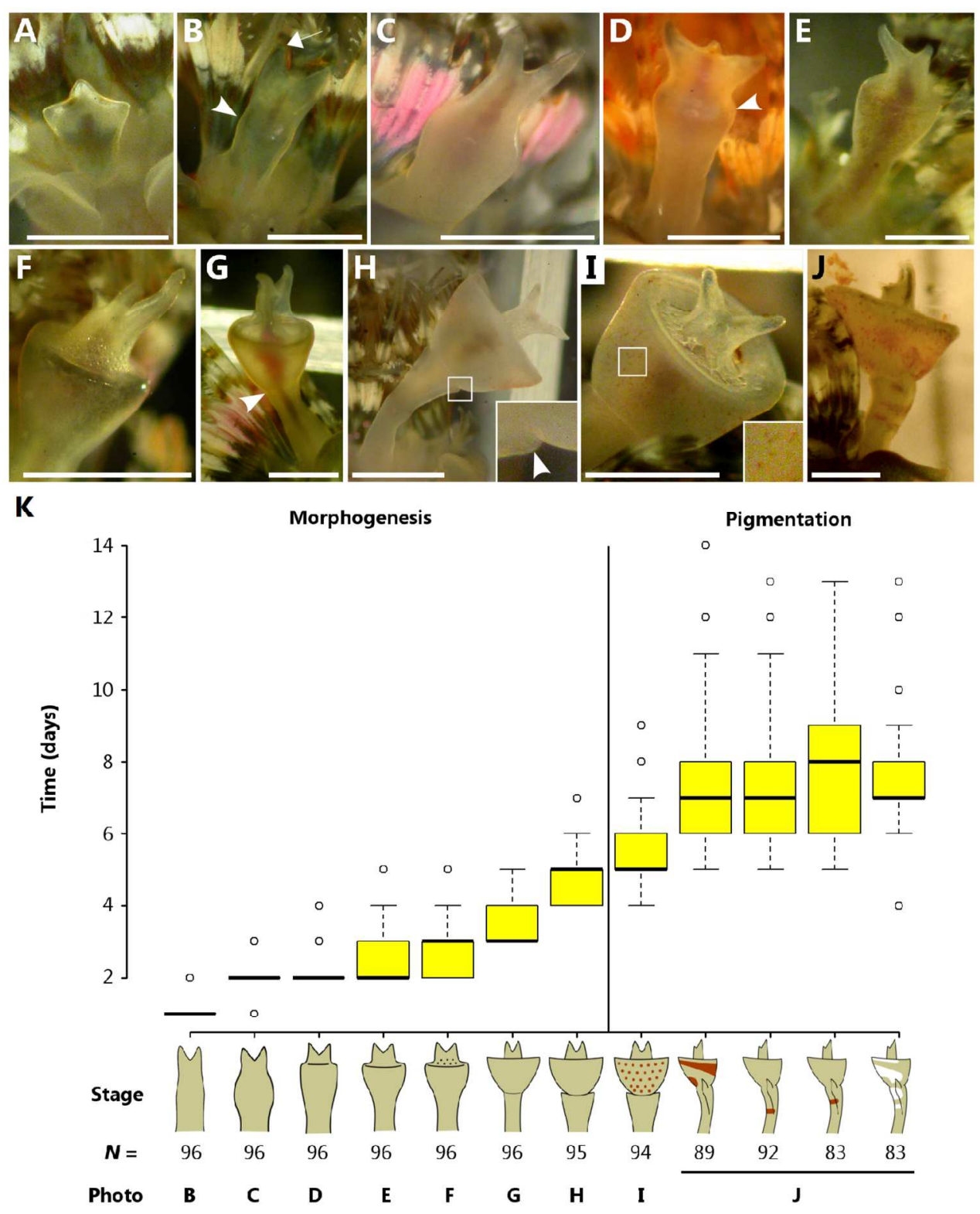

$164 \times 200 \mathrm{~mm}(300 \times 300$ DPI $)$ 


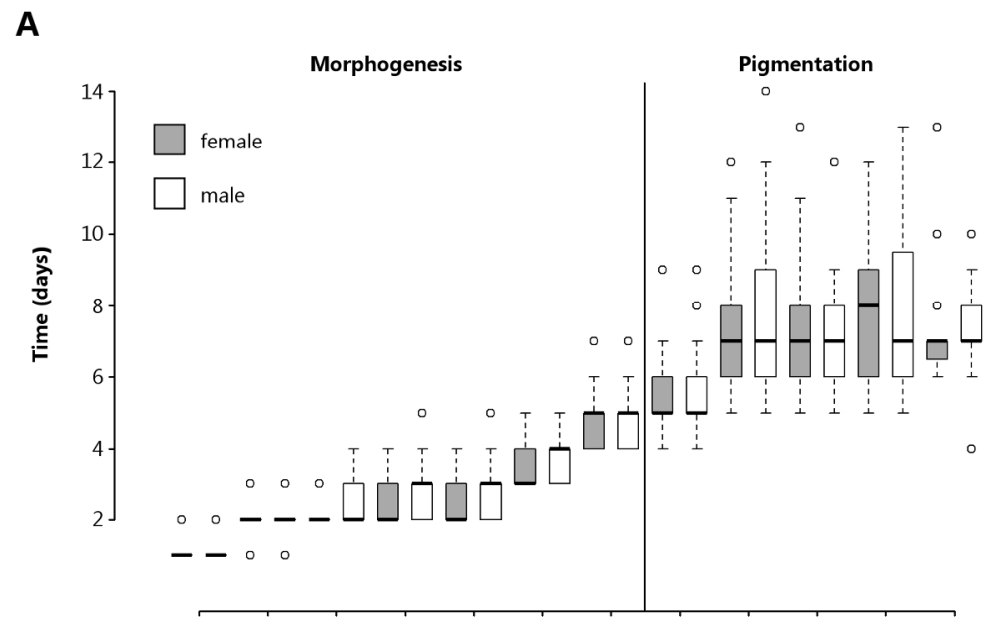

B

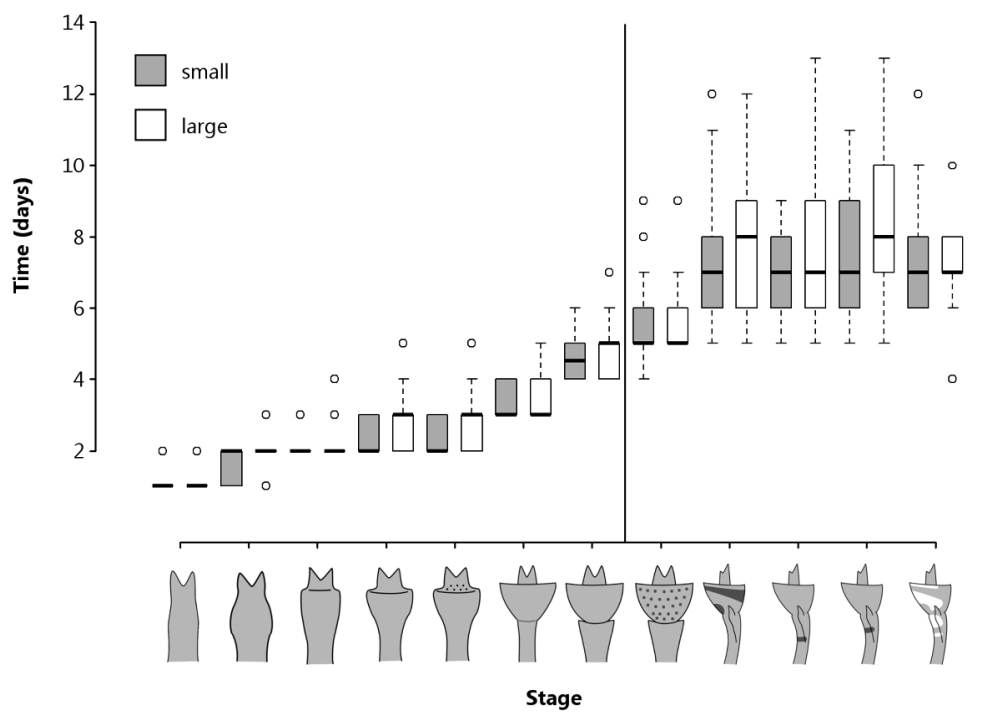

$160 \times 220 \mathrm{~mm}(300 \times 300$ DPI) 


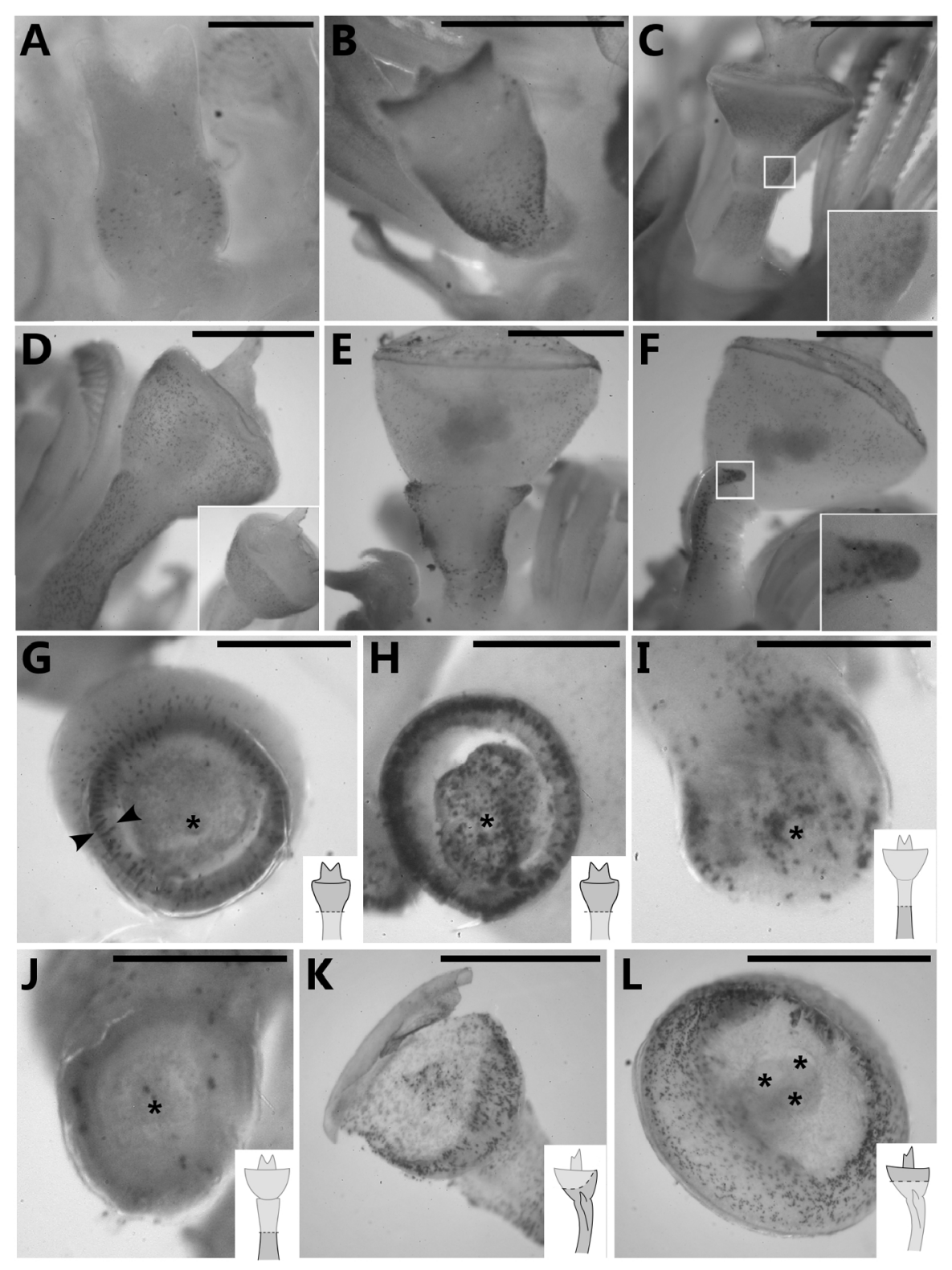

$133 \times 179 \mathrm{~mm}(300 \times 300 \mathrm{DPI})$

John Wiley \& Sons 

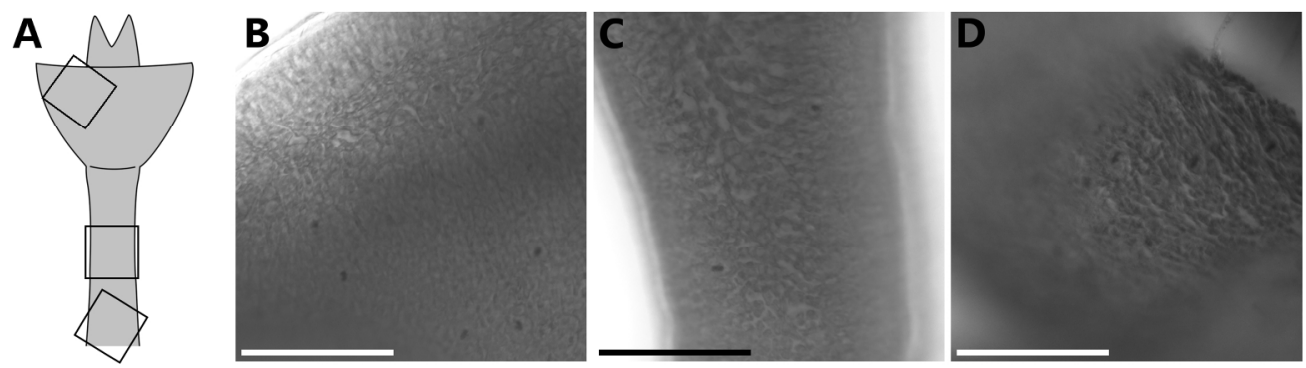

$188 \times 52 \mathrm{~mm}(300 \times 300 \mathrm{DPI})$ 

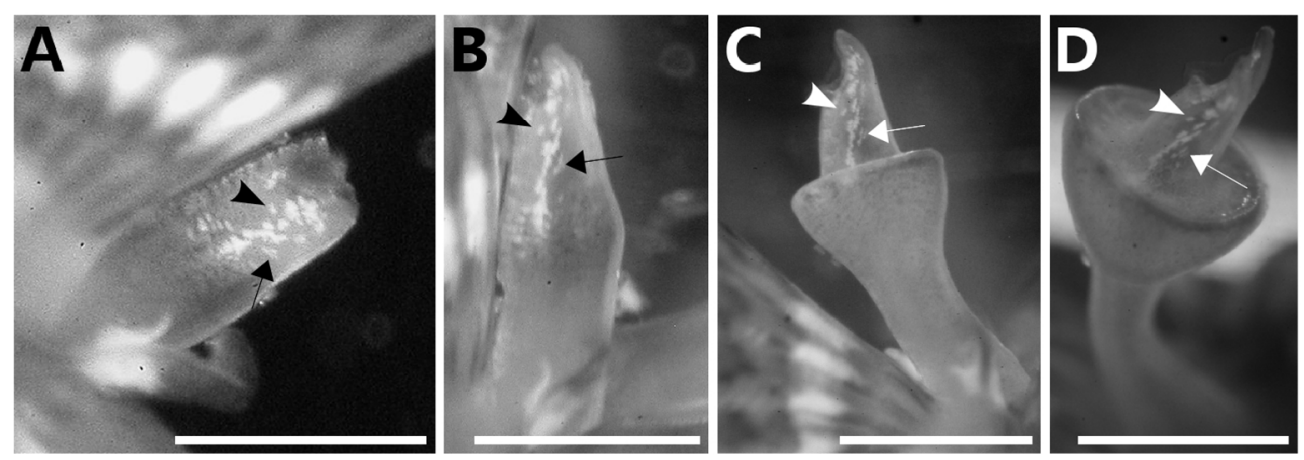

$133 \times 46 \mathrm{~mm}(300 \times 300$ DPI $)$ 


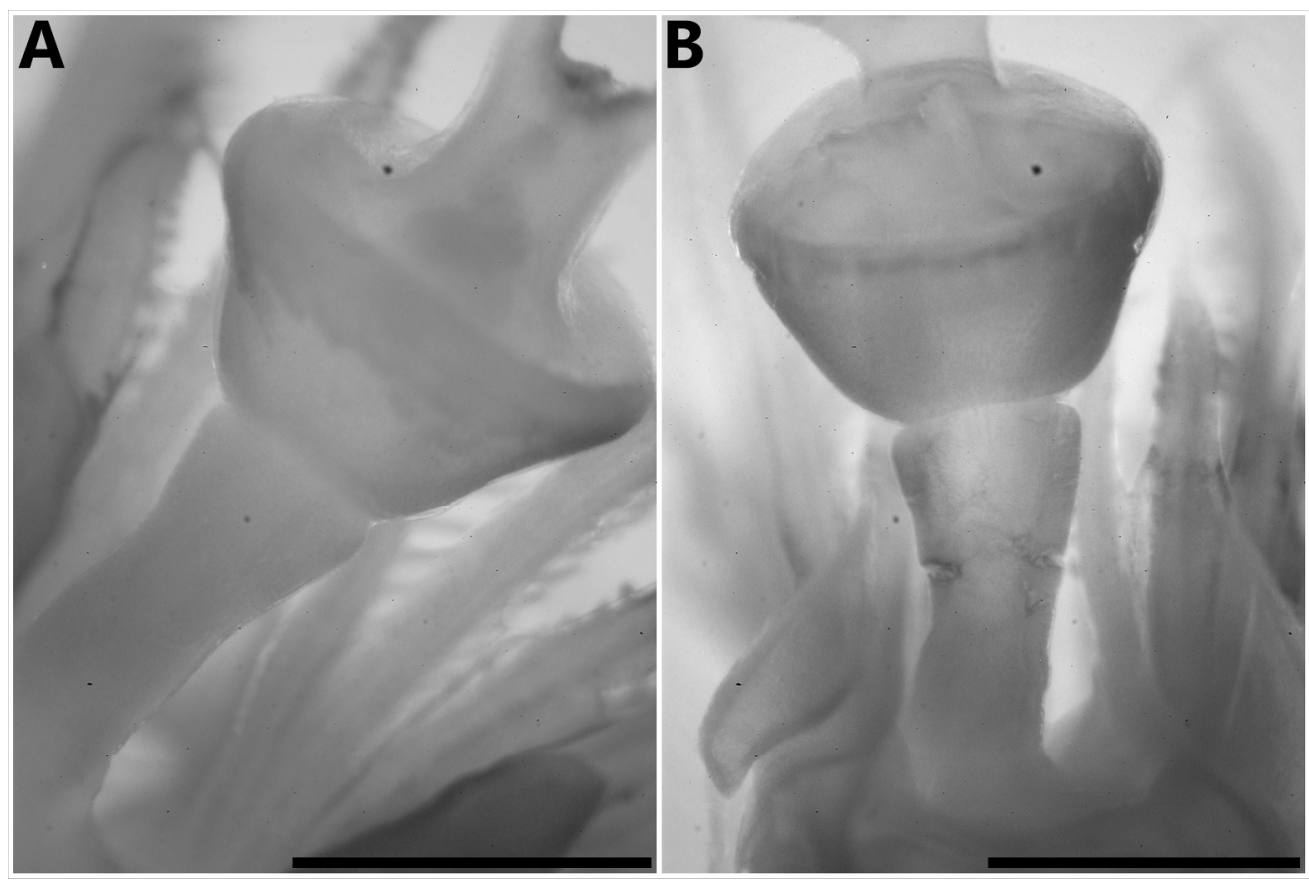

$209 \times 139 \mathrm{~mm}(300 \times 300$ DPI $)$ 